\title{
976 - Visualizing Hidden States and Spin Dynamics Using Ultrafast Electron Phase Microscopy
}

Xuewen $\mathrm{Fu}^{1}$, Myung-Geun Han ${ }^{1}$, Chunguang Jing ${ }^{2}$, Ao Liu ${ }^{2}$, Eric Montgomery ${ }^{3}$, K. Schliep ${ }^{3}$, June Lau ${ }^{3}$ and Yimei $\mathrm{Zhu}^{1}$

${ }^{1}$ Brookhaven National Laboratory, Upton, New York, United States, ${ }^{2}$ Euclid Techlabs LLC, Bolingbrook, Illinois, United States, ${ }^{3}$ National Institute of Standards and Technology, Gaithersburg, Maryland, United States

Ultrafast electron microscopy has been identified by DOE/BES as one of the major research priority directions. It provides tremendous research opportunities but has many challenges for science and technology. For instance, magnetic quasi-particles such as Majorana fermions, Skyrmions and topological vortices are promising candidates for quantum information science and technology. In vortices, various spin states associated with chirality and/or polarity can be manipulated by controlling their relaxation dynamics upon excitation. Comparing to the vortex switching through the core gyrotropic motion driven by external magnetic fields, spin-polarized currents, or spin waves, the optical excitation has advantages due to the absence of gyrotropic motion. Here we report the study of transient spin phenomena using ultrafast phase microscopy via in-situ optical quenching in spin vortex systems. We show how spin structures can be manipulated by femtosecond (fs)-laser-pulse and "hidden" spin states and switching dynamics can be revealed. behavior confined in Permalloy (Py) disks. These "hidden states" consist of a number of unexpected topological defects, vortex and antivortex, that strictly conserve the topological winding number, indicating the direct impact of the topological invariant on the magnetization dynamics in these Py disks. Furthermore, the unique spin configurations show high mirror- or rotation-symmetry because of their geometrical confinement [1].

We will also report our development of a novel laser-free electron pulser device that has been recently installed in our dedicated Lorentz microscope. The development was in collaboration with Euclid TechLab and NIST under Federal SBIR grants. The development has won the 2019 R\&D 100 Award and been granted three US patents for its novelty. The device is based on an electromagnetic-mechanical modulator that consists of two transverse deflecting cavities, three magnetic quadrupoles, and a variable tiny beam aperture [2]. The device modulates and chops the incoming continue-wave $(\mathrm{CW})$ electron beam and transforms it into pico- and sub-picosecond electron pulse sequences, or pulse trains, at an RF repetition rate. The temporal pulse length and rep-rate for the device can be continuously tuned over a wide range with repetition rates from $0.05 \mathrm{GHz}$ up to $12 \mathrm{GHz}$ and a tunable bunch length from tens of ns down to 10ps. The Pulser can be routinely operated in (1) CW mode (i.e. convention TEM operation mode as the microscope itself is not modified, where the electrons from the emission cathode randomly arrive at the sample without resolvable time information), (2) stroboscopic (pump-probe) mode, and (3) pulsed beam mode for radiation sensitive materials. Instrument performance on imaging and diffraction using the pulsed beam will be compared with the continued electron beam. Preliminary results using the RF excitation on electromagnetic wave propagation, metal-insulation-transition and magnetic quasi-particles including topological spin chiral structures and their switching behavior will be presented [3]. 


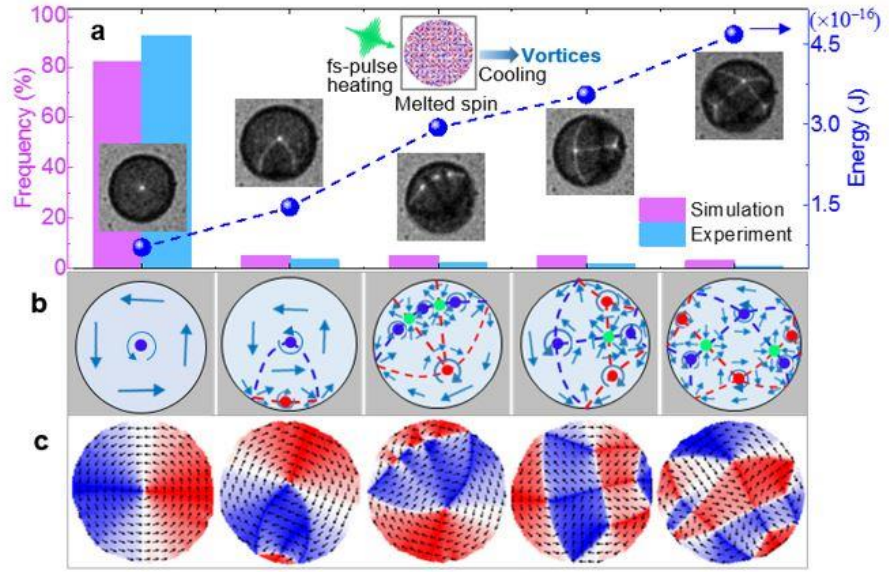

Figure 1. Optical manipulation of spin vortex visualized by ultrafast electron phase microscopy. Selected magnetic structures in Permalloy circular disks after the fs-laser pulse excitation (fluence of $12 \mathrm{~mJ} / \mathrm{cm} 2$ ) and quenching at a cooling rate up to $1012 \mathrm{~K} / \mathrm{s}$. (a-c) Statistic occurrence frequency distribution and energy evolution of experimentally observed magnetic structures (a-b) along with the micromagnetic simulations (c) of vortex and antivortex pairs, revealing the "hidden states" that have not been seen in traditional fieldand current-induced spin switching processes. Red and blue colors in (b) and (c) correspond to opposite spin directions.

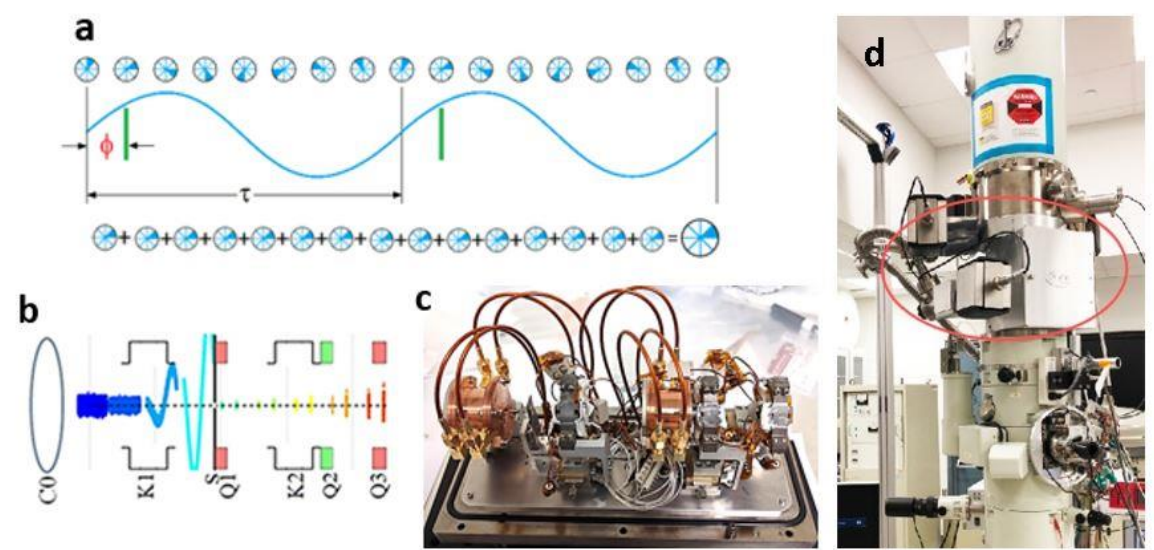

Figure 2. (a) Principle of the stroboscopic imaging with the beam chopper. Top circles represent the phase change of a continue beam while bottom ones the selection of electrons in phase-space using a tiny chopping aperture. (b) Calculated beam trajectory in the pulser from continued electron beam to pulsed electron beam. C0: condenser lens, K: beam kicker (transverse deflecting cavity), Q: quadrupole lens. (c) The actual pulser device that is retrofit into a commercial TEM and covert a traditional TEM to a laserfree ultrafast stroboscopic microscope. (d) The Pulser, marked by the red circle, is installed on the Lorentz microscope (JEOL 2100F-LM) for electric RF excitation and stroboscopic ultrafast phase microscopy. The pulser frequency is tunable, from $0.05-12 \mathrm{GHz}$, which corresponds to the time scale of tens of ns to $10 \mathrm{ps}$. The Pulser can be routinely operated in (1) Conventional TEM mode where the electrons randomly arrive at the sample without resolvable time information, (2) stroboscopic (pumpprobe) mode, and (3) pulsed beam mode for radiation sensitive materials. 


\section{References}

[1] Fu, X., et al., "Optical manipulation of magnetic vortices visualized in situ by Lorentz electron microscopy", Sci. Adv. 4, eaat3077, 1-11 (2018).

[2] Jing, C., et al., "Tunable electron beam pulser for picoseconds stroboscopic microscopy in transmission electron microscopes", Ultramicroscopy 207, 112829, 1-4 (2019).

[3] Work at BNL was supported by the US DOE-BES, MSED, under Contract No. DESC0012704. 